\title{
Recuperación de ácidos y metales en baños agotados del decapado de aceros inoxidables
}

\author{
C. $\operatorname{Frías}^{(*)}$ y O. Pérez ${ }^{(*)}$
}

\begin{abstract}
Resumen En la operación del decapado de los aceros inoxidables se producen anualmente en Europa unos $300.000 \mathrm{~m}^{3} /$ año de efluentes de baños agotados. El tratamiento normal de estos efluentes mediante neutralización y depósito de los lodos generados presenta graves inconvenientes que requieren ser subsanados, para lo cual se ha comenzado a desarrollar un nuevo proceso denominado proceso PIBARE, que presenta una gran potencialidad comercial basado en el análisis técnico-económico preliminar para una planta de $10.000 \mathrm{~m} 3 / \mathrm{año}$, donde se obtiene un "pay back period" de unos dos años, con otras ventajas medioambientales, ya que se reciclarían los ácidos libres y complejos y se recuperarían los metales como subproductos comerciales o aleaciones reciclables. Se presenta una visión general del proyecto PIBARE y los resultados obtenidos en la etapa de laboratorio que se desarrolla actualmente..
\end{abstract}

Palabras clave: Decapado. Acero inoxidable. Ácidos. Recuperación. Metales.

\section{Acids and metals recovery from spent pickling baths of stainless steels}

\begin{abstract}
During the pickling of stainless steels around $300.000 \mathrm{~m}^{3} / \mathrm{year}$ of effluents from spent baths are produced in Europe. The usual treatment of these effluents by neutralisation and slurry disposal gives important disadvantages which are necessary to solve. A new process is being developed, called PIBARE, which shows a good future market in base of a preliminary technical-economical study for a plant of $10.000 \mathrm{~m}^{3} / \mathrm{year}$, where a pay-back period of two years is obtained. Furthermore, other environmental advantages are produced, as free and complex acids recycling and metals recovery as commercial by-products or recycled alloys. This article shows a general review of PIBARE project and the results obtained in the laboratory step which is been developed at the moment.
\end{abstract}

Keywords: Pickling. Stainless steel. Acids. Recovery. Metals.

\section{INTRODUCCIÓN}

El decapado del acero inoxidable es una operación química que se realiza por inmersión en baños ácidos para eliminar la capa de óxidos formada sobre la superficie del acero inoxidable después de los tratamientos térmicos. En general, existen dos tipos de baños para realizar el decapado: ácido nítrico + ácido fluorhídrico (1), y ácido sulfúrico + agua oxigenada + ácido fluorhídrico (2). Actualmente, más del $90 \%$ de las plantas de decapado usan los baños de tipo nítrico-fluorhídrico, si bien por razones ambientales van avanzando paulatinamente los de tipo sulfúrico-fluorhídrico.

(*) Centro de I+D de Técnicas Reunidas. Sierra Nevada, 16. 28830-San Fernando de Henares (Madrid, España).
Durante la operación de decapado, los ácidos se van consumiendo y los metales, disolviéndose en el baño. El hierro es el elemento dominante seguido por el cromo y el níquel. En consecuencia, se produce un incremento de la concentración de metales en la solución ácida y ante la posibilidad de que precipiten los fluoruros de hierro, el baño se va sustituyendo parcialmente, drenando parte de su contenido y reponiendo ácidos nuevos.

Los efluentes procedentes de los baños de decapado representan un grave problema medioambiental al tratarse de un residuo que contiene nitratos, fluoruros, ácidos y un elevado contenido de metales pesados. El método usual de tratar estos baños es neutralizar con lechada de cal, con lo que se producen unos lodos que se envían a un depósito.

Aparte los graves problemas medioambientales, aparecen también importantes deficiencias en la 
operación de decapado debido a que las condiciones operativas en los baños no son estables y cada cierto tiempo se requiere parar para limpiar y ajustar su composición.

Tomando como base la información aportada por los productores europeos de acero inoxidable y aceros aleados, el volumen total de baños agotados producido en Europa es de $300.000 \mathrm{~m}^{3} / \mathrm{año} \mathrm{y}$ el residuo acumulado tras la neutralización se estima en unas 150.000 t/año, para una producción total de acero inoxidable de 6.000 .000 t/año.

Tras el análisis de la situación actual, se ha detectado la necesidad de desarrollar un nuevo proceso integrado para tratar los baños agotados del decapado de acero inoxidable tratando de resolver los problemas existentes o reducir su importancia. El nuevo proceso debe tratar de eliminar el problema de la generación de residuos y a la vez regenerar y reciclar los ácidos así como recuperar los metales contenidos en el efluente, obteniendo subproductos comerciales valiosos que contribuyan a su viabilidad económica.

\section{PROCESOS ACTUALES DE TRATAMIENTO}

Durante los últimos años, se ha realizado un importante esfuerzo investigador encaminado a la regeneración de ácidos, tanto por razones económicas como buscando unas más estables condiciones de operación. De este modo, han surgido una serie de procesos comerciales que regeneran los ácidos del baño, siendo de destacar los siguientes.

Resinas de retención de ácidos: Permiten recuperar los ácidos libres usando un tipo de resina que retienen los ácidos y dejan pasar los complejos metálicos (3). Los ácidos recuperados se reciclan al baño, mientras que la disolución que contiene los metales se neutraliza normalmente, lo que produce lodos que se envían a un depósito.

Diálisis: Es una técnica desarrollada fundamentalmente en Japón (4), pero que va ganando aplicación en Europa. Igual que en el caso de las resinas, recupera sólo los ácidos libres. Por tanto, en este caso, la neutralización también se hace necesaria.

Procesos basado en electrodiálisis: La recuperación de ácidos libres además de ácidos complejados es posible por medio de procesos desarrollados en Estados Unidos (5) y en Japón, basados en la neutralización del baño con potasa, que, a su vez, se regenera por electrodiálisis y se recicla, de manera que el coste principal del proceso es energía eléctrica. No se evita, sin embargo, la generación de lodos de hidróxidos metálicos contaminados que deben enviarse al vertedero. El elevado coste de inversión, así como la no recuperación de los metales son sus principales inconvenientes.

Evaporación: Este tipo de proceso se usa comercialmente en Europa (6) y permite la recuperación tanto de los ácidos libre como complejados. Este proceso requiere también una elevada inversión y presenta limitaciones para recuperar los metales.

\section{NUEVO PROCESO PROPUESTO (PROCESO PIBARE)}

\subsection{Objetivos}

Incrementar la eficacia del decapado de acero inoxidable, manteniendo condiciones operativas estables, y combinando ventajas económicas con un mayor cuidado del medioambiente, es decir, una reducción sustancial de costos en la compra de los ácidos, reciclando todos los ácidos del baño, y una máxima recuperación de metales valiosos, junto con la no emisión de aguas contaminadas ni sólidos residuales.

\subsection{Desarrollo del proceso PIBARE}

El proceso PIBARE intenta superar las limitaciones de los actuales sistemas de tratamiento de los baños agotados, según los objetivos planteados, para lo cual se propone la combinación de cuatro etapas principales (Fig. 1):

- Recuperación de ácidos

- Separación de metales y obtención de subproductos

- Recuperación de componentes químicos

- Tratamiento de aguas de lavado

Recuperación de ácidos: Como una nueva aproximación, se va a trabajar utilizando la técnica de extracción con disolventes basándose en la última generación de extractantes disponibles comercialmente ( 7 y 8 ).

Se va a llevar a cabo un estudio técnico-económico de las diferentes tecnologías y se seleccionará una ruta final para recuperar ácidos. El líquido sin ácido libre se tratará para recuperar los metales en las siguientes etapas.

Separación de metales: Para lograr una separación de metales eficiente se aplicarán nuevas técnicas:

- Precipitación catalítica selectiva: Nuevo proceso mediante el cual es posible separar los diversos metales mientras éstos son precipitados. 


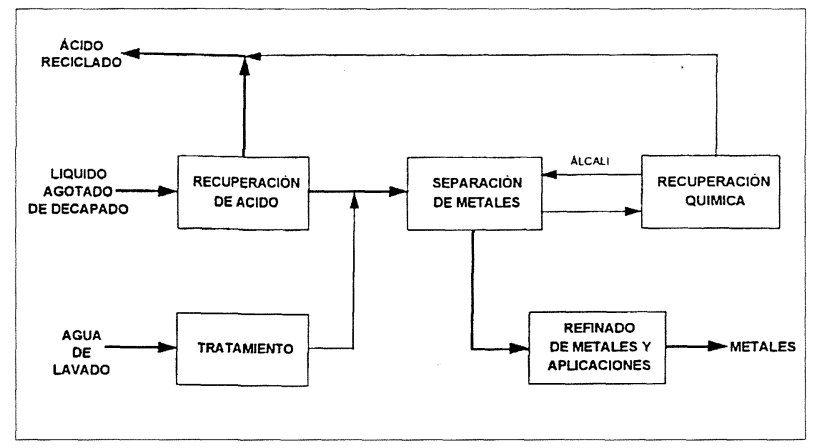

FIG. 1.- Diagrama conceptual proceso PIBARE.

FIG. 1.- PIBARE process conceptual diagram.

- Electrodeposición: Nuevo modelo de celda electrolítica capaz de electrodepositar aleaciones de metales y regenerar ácidos.

Los metales recuperados pueden ser directamente reciclables o requerir, en algún caso, una purificación o refinado para obtener un subproducto comercial.

Recuperación química: Esta etapa permite la regeneración de los ácidos complejos y residuales después de la separación de metales. Se va a desarrollar un nuevo proceso basado en el uso de membranas monopolares, porque los actuales procesos de membrana, que utilizan las bipolares, no pueden producir la concentración de ácido deseada en torno al $20-30 \%$.

Tratamiento del agua de lavado: Aplicación de las nuevas tecnologías de separación, basadas en membranas especiales, que se están desarrollando y que permiten la separación de ácidos y metales, produciendo un líquido vertible.

\subsection{Aspectos innovadores del proceso PIBARE}

Tomando como base lo expuesto en relación con las limitaciones de los procesos actuales y con los objetivos planteados para desarrollar el nuevo proceso, se pueden destacar los siguientes aspectos innovadores, que, a modo de resumen, se presentan en la tabla I:

- Desarrollo de un nuevo proceso integrado que eliminará la producción actual de residuos tóxicos y permitirá una máxima recuperación y reciclado de los ácidos.

- Desarrollo de una tecnología novedosa, basada en la oxiprecipitación catalítica o en la electrodeposición de aleaciones, que permitirá recuperar y valorizar los metales.
TABLA I.- Comparación entre el proceso PIBARE y otros procesos

TABLE I.- Comparison between PIBARE process and other processes

\begin{tabular}{|c|c|c|}
\hline CONCEPTO & $\begin{array}{c}\text { Procesos } \\
\text { Actuales }\end{array}$ & $\begin{array}{c}\text { Proceso } \\
\text { PIBARE }\end{array}$ \\
\hline $\begin{array}{c}\text { Recuperación } \\
\text { de ácido }\end{array}$ & Ácido libre & $\begin{array}{c}\text { Ácido libre } \\
\text { y complejado }\end{array}$ \\
\hline $\begin{array}{c}\text { Recuperación } \\
\text { de metales }\end{array}$ & $\begin{array}{c}\text { Normalmente } \\
\text { ninguna }\end{array}$ & Total \\
\hline Residuos sólidos & $\begin{array}{c}\text { Lodos de } \\
\text { neutralización }\end{array}$ & No se producen \\
\hline Efluentes líquidos & Ácidos agotados & No se producen \\
\hline $\begin{array}{c}\text { Impacto } \\
\text { medioambiental }\end{array}$ & Residuos a tratar & No existen \\
\hline $\begin{array}{c}\text { Subproductos } \\
\text { vendibles }\end{array}$ & No & Sí \\
\hline
\end{tabular}

- Desarrollo de una nueva aplicación de membranas monopolares para reciclar ácidos.

- Aplicación de las nuevas tecnologías de separación por membranas para eliminar bajas concentraciones de metales pesados y ácidos de las aguas de lavado.

\subsection{Datos económicos preliminares}

La cantidad total de baños agotados procedentes del decapado de acero inoxidable en Europa es de unos $300.000 \mathrm{~m}^{3} /$ año. Basándose en la composición de los baños y en el valor económico de ácidos y metales potencialmente recuperables, se podría obtener un ahorro anual de unos 120 millones de ECU para la industria europea del acero inoxidable.

Tomando como base los conocimientos actuales del proceso PIBARE, se ha realizado una estimación preliminar para una planta de tratamiento de $10.000 \mathrm{~m}^{3} /$ año. El resultado es que será precisa una inversión de 4,5 millones de ECU y un coste aproximado de explotación de 2,5 millones ECU/año, que incluye el $25 \%$ del costo de la inversión para intereses y devaluaciones, así como mantenimiento, reactivos, consumo y mano de obra. Teniendo en cuenta que se recuperan los ácidos por un valor de 2,5 millones de ECU/año y los metales, con un valor de 1,6 millones ECU/año, y que se consigue, además, un ahorro en los costes de neutralización y de depósito de los efluentes actuales, estimado en 1,1 millones ECU/año, se obtendría un cash flow positivo de 2,7 millones ECU/año, con un pay-back period para la nueva planta de 2 años, aproximadamente. 


\section{ESTADO ACTUAL DEL PROYECTO PIBARE}

El proyecto PIBARE está en su primer año de desarrollo, teniendo previsto un programa de tres años de duración, que incluirá las siguientes etapas:

- Desarrollo de las etapas principales a nivel de laboratorio

- Integración y redefinición del proceso global

- Diseño, montaje y operación de una planta piloto en continuo

- Estudio de viabilidad de un caso base industrial

En los momentos actuales se está trabajando en el desarrollado a escala de laboratorio de las principales etapas del proceso, que se va a continuar hasta finales de 1998. Los resultados preliminares más significativos obtenidos, se presentan seguidamente.

\subsection{Recuperación de ácidos}

\subsubsection{Evaporación}

Se van a estudiar dos tipos de evaporación, con y sin cristalización de sales en el fondo del evaporador. En el estudio inicial, se ha comparado la evaporación de ácido nítrico y ácido fluorhídrico en presencia o no de ácido sulfúrico. Los resultados indican que cuando no hay ácido sulfúrico sólo se evapora un $20 \%$ de los ácidos, mientras que cuando hay sulfúrico, por efecto del desplazamiento de los complejos y sales metálicas, es posible evaporar por encima del $90 \%$ de los ácidos. Si la concentración de sulfatos está en el entorno 4-5 mol/L aparece sólido precipitado en forma de sulfatos metálicos.

\subsubsection{Extracción con disolventes}

Se han seleccionado dos familias de extractantes como las más favorables para extraer ácidos. Se trata de organofosfinas y aminas terciarias (7). Los ensayos preliminares de extracción líquido-líquido muestran que los tres tipos de ácidos: nítrico, fluorhídrico y sulfúrico se pueden extraer con eficacias superiores al 90-95 \%. En los baños con presencia de varios ácidos, se demuestra una tendencia preferente inicialmente a la extracción de los ácidos fuertes, el nítrico y el sulfúrico, seguida, a continuación, de la extracción del ácido débil, fluorhídrico. En cuanto al comportamiento de los diferentes tipos de extractantes probados, se aprecia una mayor eficacia y un mejor comportamiento de las organofosfinas frente a las aminas terciarias.

\subsection{Separación de metales}

\subsubsection{Oxiprecipitación catalítica de metales}

Se pretende separar los metales de forma individual, obteniendo compuestos de óxidos o hidróxidos que sean reciclables o vendibles como subproductos.

En los ensayos preliminares se ha utilizado hidróxido amónico a $45^{\circ} \mathrm{C}$ con una circulación de aire de $10 \mathrm{~L} / \mathrm{min}$. Los sólidos se recuperaron a $\mathrm{pH}$ 1,5 y 6,0 observándose que ácidos inorgánicos libres están presentes en el sólido, principalmente ácido nítrico. La fase cristalina del sólido filtrado a pH 1,5 está hecha de molibdatos y nitratos de hierro principalmente, con algo de magnetita y oxohidróxidos de hierro del tipo $\mathrm{FeOOH} \delta$. La fase cristalina del sólido encontrado a pH 6,0 está formada por cromatos de hierro hidratados y oxohidróxidos de hierro del tipo $\mathrm{FeOOH} \delta$.

\subsubsection{Electrodeposición de metales}

Se comenzaron los estudios realizando ensayos de ciclovoltametría y caracterizando las reacciones electroquímicas y determinando su mecanismo y constantes cinéticas. Posteriormente, se ha comenzado el estudio de electrodeposición de metales utilizando microceldas de laboratorio y centrándose fundamentalmente en la reacción catódica. Los resultados preliminares sobre la electrólisis de hierro y de hierro + níquel, según un planteamiento factorial de tipo $2^{\mathrm{n}}$, tomando como variables: $\mathrm{pH}$, temperatura, densidad de corriente y concentración de metal, muestran que el $\mathrm{pH}$ es la variable que presenta mayor efecto sobre la calidad del depósito y eficacia de corriente. Así, a $\mathrm{pH}=1$, la eficacia obtenida es de un $60 \%$, mientras que a $\mathrm{pH}=3$ se obtienen eficacias de corriente del $100 \%$, trabajando a densidades de corriente de $400 \mathrm{~A} / \mathrm{m}^{2}$.

\subsection{Recuperación de componentes químicos}

Se han comenzado las pruebas de electrodiálisis con soluciones sintéticas tratando de ajustar el banco de pruebas a las condiciones del proceso actual. Los ensayos preliminares están centrados en las pruebas y selección de diferentes tipos de membranas comerciales, siendo los parámetros de control: el voltaje, la eficacia de separación de los componentes y el comportamiento físico y vida de las membranas.

\subsection{Tratamiento de las aguas de lavado}

El programa experimental previsto incluye la comparación de diferentes técnicas basadas en 
separación por membranas: ósmosis inversa, ultrafiltración y nanofiltración. Los resultados iniciales muestran que la ósmosis inversa permite una separación eficiente de los iones, dejando pasar una solución no contaminada, si bien se requiere de una experimentación más detallada para seleccionar las membranas adecuadas para un medio ácido y oxidante como es el que nos ocupa.

\section{CONCLUSIONES}

El consorcio que dirige el proyecto PIBARE ha detectado la necesidad, a nivel europeo, de desarrollar un nuevo proceso para recuperar y reciclar los subproductos generados en el decapado de acero inoxidable para evitar los problemas relacionados con el medio ambiente, ahorrar costes y mejorar la técnica del proceso de decapado.

La estimación económica preliminar y la comparación técnica con otros procesos existentes nos indica que el proceso PIBARE presenta una gran potencialidad comercial si se consigue su desarrollo conforme a los objetivos planteados. El pay-back period para una planta que tratase $10.000 \mathrm{~m}^{3} /$ año de baños agotados sería de unos 2 años aproximadamente.

En la etapa de recuperación de ácidos, la evaporación necesita la adición de ácido sulfúrico para recuperar un $90 \%$ de los ácidos. Mientras que en la extracción las organofosfinas parecen ser mejores extractantes que las aminas terciarias, además de que los ácidos fuertes se extraen preferentemente a los débiles. La extracción de ácidos en los ensayos preliminares es superior al 90-95\%.

En la etapa de separación de metales, la oxiprecipitación catalítica nos indica que parece que, a cualquiera de los $\mathrm{pH}$ trabajados, los sólidos van acompañados de ácidos minerales en su composición, que será necesario eliminar. Mientras que en la electrodeposición de aleaciones metálicas parece posible alcanzar el $100 \%$ de eficacia si se trabaja a $\mathrm{pH}$ próximo a 3 y densidad de corriente de 400 $\mathrm{A} / \mathrm{m}^{2}$.

Se han comenzado los ensayos de electrodiálisis para la recuperación de componentes químicos, habiéndose centrado en las pruebas de selección de membranas.

En la etapa de tratamiento de las aguas de lavado, la ósmosis inversa se presenta prometedora, si bien es necesario seguir trabajando para seleccionar la membrana más adecuada a este medio. Se está pendiente de los resultados de aplicación de otras técnicas, como es la nanofiltración.

\section{Agradecimiento}

Los autores agradecen a la Comunidad Europea el apoyo que está prestando en el desarrollo del proyecto PIBARE, que está siendo parcialmente financiado a través del programa BRITE-EURAM.

Igualmente, los autores de este artículo agradecen la autorización para su publicación del consorcio que está desarrollando el proceso PIBARE, formado por: TÉCNICAS REUNIDAS, Universidad Complutense (Dpto. de Ingeniería Química), CENIM (CSIC), TNO, VECOM, OUTOKUMPU STEEL, HERAEUS y ACCIAI SPECIALI TERNI.

\section{REFERENCIAS}

(1) Rydberg, J., Reinhardt, H., Lunden, B.P., y Haglund, P. Intern. Symp. on Hydrometallurgy, AIME, Chicago. Nueva York (EE.UU.), 1973: 589-611.

(2) Fortunati, S., Novaro, C., Pedrazzini, A. y Pollastrelli, A. Enviromental friendly process for stainless steel pickling. Innovation stainless steel. Florencia (Italia). Oct. (1993).

(3) BRown, C.J. Productivity improvements through recovery of pickle liquors with the APU process. Iron Steel Eng. Ene. 1990

(4) Información técnica de los principales productores de membranas y equipos de electrodiálisis. (Aqualytics (EE.UU.), Tokuyama Soda (Alemania), Solvay (Francia), Ionics (EE.UU.), Asahi Chemical (Japón), Asahi Glass (Japón) ) 1996.

(5) Hutter-Byszewski, C. y Bogeatzs, A.S. Aquatech systems. A commercial process to recycle spent pickle liquor". AISE Year Book. 1988: 119-123.

(6) Nyman, B. y Koivunen, T. The Outokumpu process for pickling acid recovery. Iron Control Hydrometallurgy. The Metallurgical Society of CIM, Ellis Horwood Ltd. 1986: 520-536.

(7) Información técnica de los productores de extractantes capaces de recuperar ácidos. Henkel Corp. (Alemania) y Cianamid (EE.UU.) 1997.

(8) Kuylenstierna, U. y Ottertun, H. Solvent extraction of nitric-hydrofluoric acid from stainless steel pickling solutions. Proc. Int. Solvent Conf. (1974), Vol. 3, 2803-16. 